Research Article

Human and Medical Genetics

\title{
Expression of Ubiquitin-specific protease 7 in oral squamous cell carcinoma promotes tumor cell proliferation and invasion
}

\author{
Xiaojie Yang ${ }^{1,2^{*}}$, Jiamin $\operatorname{Jin}^{1 *}$ (D), Jinfeng Yang ${ }^{1,3^{*}}$, Lihua Zhou ${ }^{1}$, Sisi Mi ${ }^{1}$ and Guangying Qi ${ }^{1}$ \\ ${ }^{I}$ Guilin Medical University, Laboratory of Tumor Immunology and Microenvironmental Regulation, Guilin, \\ Guangxi, China. \\ ${ }^{2}$ Hospital of Guangxi Medical University, Department of Pathology, Nanning, Guangxi, China. \\ ${ }^{3}$ Guilin Medical University, Department of Immunology, Guilin, Guangxi, China.
}

\begin{abstract}
Oral Squamous Cell Carcinoma (OSCC) is the most common malignant cancer affecting oral cavity. Recent studies have demonstrated that Ubiquitin-specific protease 7 (USP7) was upregulated in several types of cancers. USP7 expression was associated with various proto-oncogenes and tumor suppressor genes. However, USP7 expression level and its functional role in OSCC is unclear. In the current study, we showed that USP7 expression in OSCC tissues was generally upregulated compared to normal adjacent tissues by using IHC. Furthermore, statistical analysis uncovered that USP7 expression was positively correlated with Ki-67, MMP2, VEGF in OSCC tissues. Importantly, high USP7 expression was significantly correlated with lymph node metastasis and histological differentiation in OSCC patients. So, our hypothesis is that USP7 plays a tumor-promoting role in OSCC. Knocking down of USP7 in tumor cells not only suppressed HSC3 cells proliferation, migration and invasion, but also promoted cell apoptosis. Moreover, USP7 siRNA blocked the activation of Akt/ERK signaling pathway. In conclusion, data presented here suggests that USP7 promotes the progression of OSCC. USP7 may be used as a new therapeutic target for OSCC diagnosis and treatment.
\end{abstract}

Keywords: Oral Squamous Cell Carcinoma, USP7, siRNA, proliferation, invasion.

Received: February 23, 2021; Accepted: August 30, 2021.

\section{Introduction}

Oral cancer is a type of severe malignant head and neck tumor, with about $90 \%$ oral cancer cases are Oral Squamous Cell Carcinoma (OSCC) (Bray et al., 2018). There were about 70 million patients diagnosed with OSCC worldwide in the past five years (Jason et al., 2020). The 5-year survival rate is about 39\% (Zhang et al., 2019). The prognosis of OSCC is still poor because of the lack of effective therapeutic target genes (Haddad and Shin, 2008; Wikner et al., 2014). Thus, there is urgent need to investigate the pathogenesis and molecular mechanism of OSCC occurrence and progression for further improvement of the survival and life quality.

The ubiquitin proteasome system is important for maintaining protein turnover for cancer progression (Young et al., 2019). We have previously demonstrated that USP22 was upregulated in OSCC and hepatocellular carcinoma (Tang et al., 2015; Liu et al., 2019). USP22 expression is a positively correlated with Aurora-B and Survivin expression. USP22 can also regulate the expression of cell cycle related proteins in order to promote cancer cell growth (Liu et al., 2019). Recent studies show that USP7, which is involved in tumor-genesis and process (Song et al., 2008; Zhang et al., 2016), could be used for novel drug target in cancer therapy (Zhou et al., 2018). USP7 expression is not only closely related to tumor

Send correspondence to Guangying Qi. Guilin Medical University, Laboratory of Tumor Immunology and Microenvironmental Regulation, Zhiyuan Road, Guilin, Guangxi 541004, China. E-mail: qgy@glmc. edu.cn.

*These authors contributed equally to the article. cell proliferation and invasion (Chiu et al., 2014), but also affects some critical signaling pathways (Zhou et al., 2018). However, the underlying mechanism of how USP7 affects these processes in OSCC remains unclear.

USP7, an important member in ubiquitin-specific protease family, was first discovered in 1997 (Everett et al., 1997). USP7 can regulate the expression of various tumor-related genes, such as p53 and Ki-67 (Sarkari et al., 2010; Zhou et al., 2018). USP7 promotes the proliferation of non-small cell lung cancer cell via stabilizing Ki-67 protein (Zhang et al., 2016). Knockdown of USP7 expression increases p53 expression and inhibits colon cancer cells proliferation (Colland et al., 2009). OSCC patients with high $\mathrm{Ki}-67$ expression showed a significantly increased risk of poor overall survival as well as disease-free survival (Jing et al., 2018). In addition, invasion-metastasis-related factor matrix metalloproteinase 2 (MMP2) was shown to improve OSCC migration and invasion (Celentano et al., 2021); Angiogenesis was also involved in OSCC progression. Vascular endothelial growth factor (VEGF) and its receptor VEGFR are the main factors that are responsible for angiogenesis (Mărgăritescu et al., 2009). VEGF is highly expressed and considered as a marker of OSCC progression (Mărgăritescu et al., 2010). However, the relationship of USP7 with Ki-67, MMP2, and VEGF in OSCC is still unclear.

Therefore, aim of the current study is to investigate the molecular mechanism of USP7 in regulating tumor progression and signaling pathways of OSCC and to verify its relationship with Ki-67, MMP2, and VEGF. 


\section{Material and Methods}

\section{Patient samples}

92 cases of surgical OSCC specimens were collected and sorted from the Department of Pathology in the Affiliated Hospital for Guilin Medical University (Guilin, Guangxi, China) from 2010 to 2017 . All steps conducted were following the ethical standards of the institutional and national research committee, the 1964 Declaration of Helsinki, and its later amendments or comparable ethical standards. The research was approved by Guilin Medical University (Guilin, China) Ethics Committee. Written informed consent was provided by every participant involved in this work. The complete clinical and pathological characteristics were recorded, including age, gender, tissue histological differentiation and lymph node metastasis. Before operation, the patient were confirmed with no radiotherapy or chemotherapy treatment, and all had a definite pathological diagnosis of OSCC after operation. The normal control tissues, which were more than $5 \mathrm{~cm}$ away from the tumor site of the same patients were collected from oral mucosal tissues by using naked eye.

\section{Immunohistochemical staining}

All specimens were first immersed and fixed in neutral formalin. Later tissues were embedded with paraffin, then made into $4 \mu \mathrm{m}$ thick continuous sections. The sections were routinely dew-axed and hydrated. The EDTA antigen repair solution was boiled for 2 minutes, then was allowed to cool to room temperature. The tissue sections were blocked with an endogenous peroxidase blocker at $37^{\circ} \mathrm{C}$ for $15 \mathrm{~min}$ and incubated with polyclonal anti-USP7 antibody (rabbit, cat.\# ab4080; 1:1000; Abcam, USA), monoclonal anti-Ki-67 antibody (mouse, cat.\# ZM-0166; 1:800, zsbio, China), monoclonal anti-MMP2 antibody (mouse, cat.\# ab86607; 1:1000; Abcam, USA), and monoclonal anti-VEGF antibody (rabbit, cat.\# ab155288; 1:1000; Abcam, USA) overnight at $4{ }^{\circ} \mathrm{C}$ respectively. HRP-conjugated anti-rabbit IgG or APconjugated anti-mouse IgG (cat.\# DS-0004; zsbio, China) antibodies was incubated at $37^{\circ} \mathrm{C}$ for $30 \mathrm{~min}$. DAB substrate was added and the obtained result was observed under a microscope (x 200 magnification; light microscope; Olympus BX53F). USP7, Ki-67, MMP2 and VEGF expression were graded high $(++\sim+++)$ or low $(-\sim+)$ based on the proportion of positive tumor cells (positive cells: $>50 \%$ are strongly positive $(+++) ; 20-50 \%$ are moderately positive $(++) ; 5-20 \%$ are weakly positive $(+) ;<5 \%$ are negative $(-)$.

\section{Cell culture and treatment}

Human OSCC cell lines (HSC3, HSC4, KB) were all purchased from the American Tissue Culture and Preservation Center (ATCC). HSC3 and HSC4 cells were cultured with RPMI-1640 complete medium that have $10 \%$ fetal bovine serum (FBS) and 1\% penicillin streptomycin (PS). KB cells were cultured with DMEM (10\% FBS, 1\% PS). The medium's placement condition was $37^{\circ} \mathrm{C}, 5 \% \mathrm{CO}_{2}$ for cultivation.

\section{Western Blot}

Cells were lysed by RIPA buffer for 30 min together with protease inhibitor, the samples were centrifuged at $11000 \mathrm{~g}$ for at $4{ }^{\circ} \mathrm{C} 15 \mathrm{~min}$. Twenty microgram of protein was loaded to SDS-PAGE and transferred to polyvinyl difluoride (PVDF) membrane (Bio-Rad Laboratories, USA). The membrane was subjected to primary antibodies $\left(4 \pm 0.5^{\circ} \mathrm{C}\right.$, overnight $)$ and secondary antibodies ( $1 \mathrm{~h}$ at room temperature) respectively. Primary antibodies contained USP7 (rabbit; cat.\# ab4080; 1:1000; Abcam; USA), Cyclin A (mouse; cat.\# SC-271682; 1:500; Santa Cruz Biotechnology; USA), cleaved Caspase 3 (rabbit; cat.\# 9661; 1:1000; Cell Signaling Technology; USA), Caspase 3 (rabbit; cat.\# 19677-1; 1:1000; Proteintech Group (PG); China), Bax (rabbit; cat.\# 60267-1-Ig; 1:1000; PG; China), Bcl-2 (rabbit; cat.\# 60178-1-Ig; 1:1000; PG; China), AKT(mouse; 60203-2-Ig; 1:1000; PG; China), p-AKT(mouse; 66444-1-Ig; 1:1000; PG; China), ERK(Rabbit;cat.\#112571-AP;1:1000;PG;China), p-ERK(Rabbit;cat.\#28773-1-AP; 1:5000;PG;China). The secondary antibodies contained goat anti-mouse HRP (cat.\# ZB-2305; 1:10000, zsbio, China) or goat anti-rabbit HRP (cat.\# ZB-2301; 1:10000, zsbio, China). GAPDH (mouse; cat.\# TA-08; 1:1000; zsbio; China) was used as house-keeping gene control.

\section{Knockdown of USP7 with small interfering RNA (siRNA) transfection}

Three USP7 siRNA were designed and transfected. The sequences were listed below: siRNA-USP7-Homo-603 (5'- GCAGUGCUGAAGAUAAUAATT- 3', 5' -UUAUU AUCUUCAGCACUGCTT-3'); siRNA-USP7-Homo-2033 (5' - CAUGGGAUUUCCACAAGAUTT- 3', 5'- AUCUU GUGGAAAUCCCAUGTT-3' '); siRNA-USP7-Homo-3359 (5' to 3'- GCCCGGUAAUAUGUCUCAUTT, 5'-AUGAGA CAUAUUACCGGGCTT-3'); negative control (5' -UUCUC CGAACGUGUCACGUTT-3', 5'- ACGUGACACGTTCG GAGAA- 3') (Gene Pharma, China). HSC3, HSC4, KB cells were transfected with siRNA-USP7 or negative control by using Lipofectamine ${ }^{\circledR} 3000$ Transfection reagent (Thermo Fisher Scientific, Inc.).

\section{CCK-8 assay}

Cell suspensions of the blank group, negative control group and USP7 siRNA transfection group were inoculated in 96-well plates (each well contains about 1000 cells in 200 $\mu 1$ medium). Each group has 6 replicate wells. The cells were cultured in a carbon dioxide incubator at $37^{\circ} \mathrm{C}$. Twenty $\mu \mathrm{l}$ CCK-8 solution was added for $4 \mathrm{~h}$ to each well at interval of $0 \mathrm{~h}, 24 \mathrm{~h}, 48 \mathrm{~h}$, and $72 \mathrm{~h}$ after culture respectively. OD values were measured by absorbance at $450 \mathrm{~nm}$ with microplate.

\section{Wound-healing assay}

Cell suspensions of the blank group, siRNA control group and USP7 siRNA transfection group were plated in 6 -well plate (each well contains about $5 \times 10^{5}$ cells) for $24 \mathrm{~h}$. One hundred microliter sterile pipette tips were used to scribe a line evenly in the 6-well plate, and photographs were taken at $0 \mathrm{~h}$ and $24 \mathrm{~h}$ after scratching to observe the wound-healing ability of the relative cells.

\section{Transwell migration and invasion assay}

Cell suspensions of the blank group, siRNA control group and USP7 siRNA transfection group were plated in 
Transwell chambers (24-well chambers, Corning Inc.). For migration assay, $100 \mu 1$ cell (about $5 \times 10^{4}$ cells $/ \mathrm{ml}$ with serum-free medium) suspension was plated to the upper chamer, $600 \mu \mathrm{l}$ of medium containing $20 \%$ FBS was added to the bottom chamber. The result was analyzed according to published literature (Zeng et al., 2019); For invasion assay, Matrigel (BD Biosciences) was first diluted at 1:9 ratio before it was added to the upper chamber. Cells and medium were performed the same as the migration assay. The migrated cells were stained with crystal violet $48 \mathrm{~h}$ later. Each sample was randomly counted in three fields (100x magnification) to evaluate the invasion ability of HSC 3 cells. The result was analyzed according to published literature (Zeng et al., 2019).

\section{Statistical analysis}

All experiments were performed three times under the same conditions. The experimental data were analyzed by using SPSS v. 20.0 (SPSS, Inc, Chicago, USA) and GraphPad Prism 8. The data of multiple experiments were expressed in the form of mean \pm standard deviation. The $\chi^{2}-$ test was used for the comparison of numerical data. The data were analyzed by chi-square test and Spearman correlation analysis. The $t$-test compares the differences among the groups.
$P<0.05$ indicates that the difference between the groups was statistically significant.

\section{Results}

\section{Expression of USP7, Ki-67, MMP2 and VEGF were elevated in OSCC tissues}

To clarify the role of USP7 in OSCC progression, the expression level of USP7, Ki-67, MMP2 and VEGF were examined in 92 OSCC tissues and 20 normal tissues by using immunohistochemistry staining. The expression of USP7, Ki-67, MMP2 and VEGF were mainly restricted to the basal layers of OSCC specimen (Figure 1). In contrast, there was weak expression of the above proteins in normal oral mucosa. Interestingly, the expression of these proteins was significantly elevated in poorly differentiated OSCC samples compared to well-differentiated samples (Figure 1). USP7 and Ki-67 were primarily localized in the nuclei, while MMP2 and VEGF were localized in the cytoplasm. These findings demonstrated that USP7 expression was elevated in OSCC tissues, suggesting that USP7 and its relative genes Ki-67, MMP2 and VEGF may promote the development of OCSS.

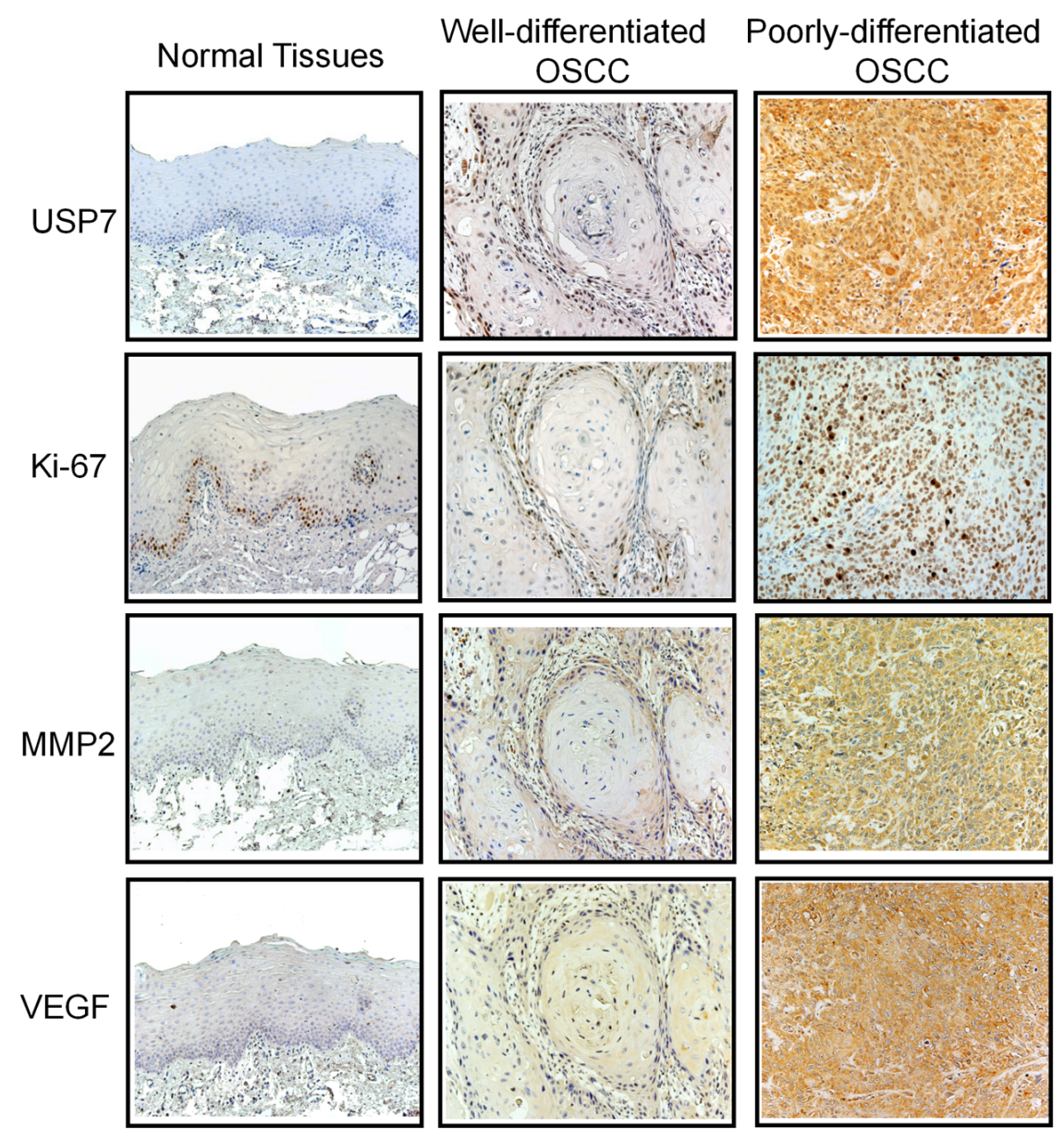

Figure 1

Figure 1 - Expression of USP7, Ki-67, MMP2 and VEGF were elevated in OSCC tissues. Staining antibodies against human USP7, Ki-67, MMP2 and VEGF to show relative protein expression in normal tissues, well-differentiated OSCC and poorly-differentiated OSCC tissues. Image magnification is 200 times. 


\section{Expression of USP7, Ki-67, MMP2 and VEGF were correlated with the clinicopathological features of OSCC}

USP7 was highly expressed in 56 OSCC cases and poorly expressed in the other 36 OSCC cases, VEGF was highly expressed in $52 \mathrm{OSCC}$ cases and poorly expressed in the other 40 OSCC cases (Table 1). The expression level of Ki-67 and MMP2 did not show significant differences in OSCC cases (Table 1). Among 56 cases with high USP7 expression, 39 cases showed high Ki-67, MMP2 and VEGF expression. Meanwhile, among 36 cases with low USP7 expression, 25 cases showed low Ki-67, MMP2 and VEGF expression (Table 2). These data indicated that USP7 expression was positively associated with Ki-67, MMP2 and VEGF expression levels.

Subsequently, USP7 expression was correlated to histological differentiation $(P<0.01)$ and lymph node metastasis $(P<0.05)$ (Table 3). High USP7 expression level was related to poorly differentiated OSCC tissues compared to well differentiated OSCC tissues (Table 3). These data suggested that USP7 expression was more prone to lymph node metastasis and poorly differentiated OSCC patients, indicating that USP7 overexpression was closely related to the malignancy of OSCC.

\section{USP7 knockdown suppresses OSCC cell proliferation and induces cell apoptosis}

In order to explore the role of USP7 in OSCC, we firstly examined USP7 expression in HSC3, HSC4 and KB cell lines by using western blot. $\mathrm{HSC} 3$ cells had the highest expression of USP7 protein (Figure 2A). So HSC3 cells were used for the following experiments. Three USP7 siRNA sequences (siRNAUSP7-603, siRNA-USP7-2033, siRNA-USP7-3359) were transfected respectively into HSC3 cells. USP7 expression was detected by western blot after transfection. Compared to the other two sequences, siRNA-USP7-603 (siUSP7) was shown to diminish USP7 expression most efficiently (Figure 2B). Thus siRNA-USP7-603 was used for the following experiments.

Next, we examined the influence of USP7 knockdown on the proliferation of OSCC cell by CCK-8 assay. The proliferation of $\mathrm{HSC} 3$ cells was significantly decreased in the USP7 siRNA group compared to blank control and siRNA control groups (Figure 2C). Cyclin A, Cyclin B and Cyclin D1 expression in HSC3 cells were reduced heavily in siUSP7 group. In contrast, the expression cyclin-dependent kinase inhibitors, p21 and p27 were increased in siUSP7 group (Figure 2D). The expression of pro-apoptotic factors

Table 1 - Differential expression analysis of USP7, Ki-67, MMP2 and VEGF in OSCC and normal tissues.

\begin{tabular}{|c|c|c|c|}
\hline & Normal Tissues & OSCC & $P$-value \\
\hline \multicolumn{4}{|c|}{ USP7 $7^{\mathrm{a}}$ expression } \\
\hline Low & 20 & 36 & \multirow{2}{*}{$<0.01$} \\
\hline High & 0 & 56 & \\
\hline \multicolumn{4}{|c|}{ Ki-67 expression } \\
\hline Low & 20 & 45 & \\
\hline High & 0 & 47 & \\
\hline \multicolumn{4}{|c|}{ MMP2 $^{\mathrm{b}}$ expression } \\
\hline Low & 20 & 45 & \\
\hline High & 0 & 47 & \\
\hline \multicolumn{4}{|c|}{ VEGF $^{c}$ expression } \\
\hline Low & 20 & 40 & \multirow{2}{*}{$<0.01$} \\
\hline High & 0 & 52 & \\
\hline
\end{tabular}

${ }^{a} U S P 7$, Ubiquitin-specific peptidase 7; ${ }^{\mathrm{b} M M P 2}$, matrix metalloproteinase 2; ${ }^{\mathrm{c}} \mathrm{VEGF}$, vascular endothelial growth factor

Table 2 - Correlation of USP7 with Ki-67, MMP2 and VEGF expression in OSCC cases.

\begin{tabular}{|c|c|c|c|}
\hline \multicolumn{4}{|c|}{ USP7 expression } \\
\hline & $\begin{array}{c}\text { Low } \\
36\end{array}$ & $\begin{array}{c}\text { High } \\
56\end{array}$ & $P$-value \\
\hline \multicolumn{4}{|c|}{ Ki-67 expression } \\
\hline Low & 33 & 12 & \multirow{2}{*}{$<0.01$} \\
\hline High & 3 & 44 & \\
\hline \multicolumn{4}{|c|}{ MMP2 expression } \\
\hline Low & 30 & 17 & \multirow{2}{*}{$<0.01$} \\
\hline High & 6 & 39 & \\
\hline \multicolumn{4}{|c|}{ VEGF expression } \\
\hline Low & 25 & 17 & \multirow{2}{*}{$<0.01$} \\
\hline High & 11 & 39 & \\
\hline
\end{tabular}


Table 3 - USP7 expression and its correlation with clinicopathological features in OSCC cases.

\begin{tabular}{|c|c|c|c|}
\hline \multicolumn{4}{|c|}{ USP7 expression } \\
\hline & $\begin{array}{c}\text { Low } \\
36\end{array}$ & $\begin{array}{c}\text { High } \\
56\end{array}$ & $P$-value \\
\hline \multicolumn{4}{|c|}{ Age (years) } \\
\hline$\geqq 50$ & 27 & 43 & \\
\hline$<50$ & 9 & 13 & \\
\hline \multicolumn{4}{|l|}{ Sex } \\
\hline Male & 18 & 48 & \\
\hline Female & 18 & 8 & \\
\hline \multicolumn{4}{|c|}{ Lymph node metastasis } \\
\hline Negative & 27 & 31 & \multirow{2}{*}{$<0.05$} \\
\hline Positive & 9 & 25 & \\
\hline \multicolumn{4}{|c|}{ Histological differentiation } \\
\hline Poor & 0 & 24 & \multirow{2}{*}{$<0.01$} \\
\hline Well & 36 & 32 & \\
\hline
\end{tabular}

A

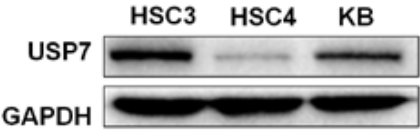

C

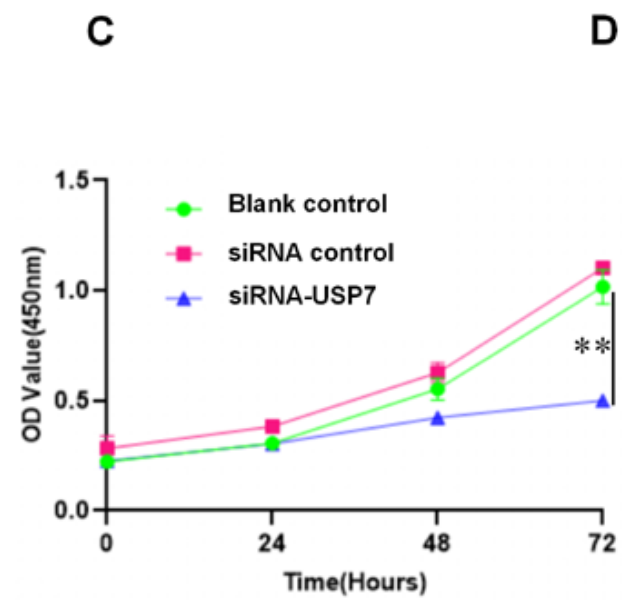

D
B

$128 \mathrm{kDa} \quad$ USP7

$36 \mathrm{kDa}$ GAPDH

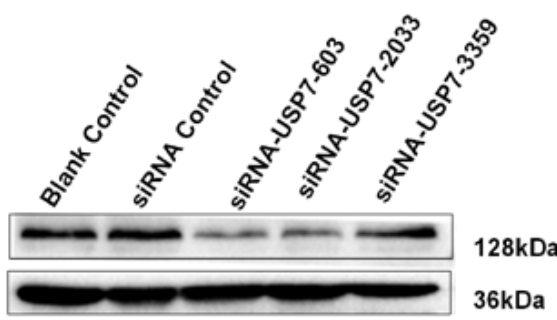

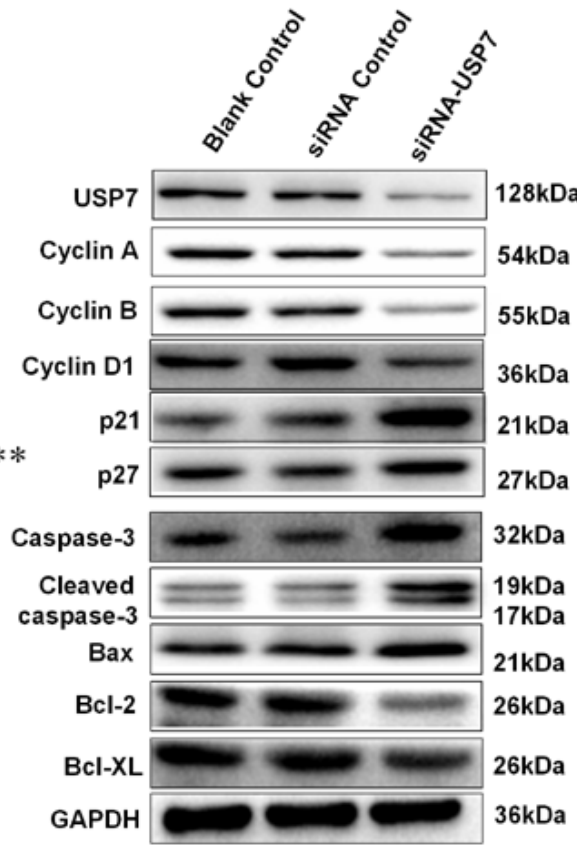

Figure 2

Figure 2 - USP7 promotes OSCC cell proliferation by affecting cell cycle-related and apoptosis-related proteins. A. Western blot was used to detect the expression level of USP7 in HSC3, HSC-4, and KB cells. GAPDH was used as control. B. USP7 expression in HSC3 cell that was transfected with USP7 siRNA or siRNA negative control, and blank control. GAPDH was used as house-keeping control. C. Growth curve for HSC3 cells with siUSP7 treatment. Forty-eight hours after USP7 siRNA treatment, 1000 cells were seeded onto 96 -well plate. Cell number at $24 \mathrm{~h}$ was set as $0 \mathrm{~h}$. Cell numbers were continuously counted at $24 \mathrm{~h}, 48 \mathrm{~h}, 72 \mathrm{~h} . * * P<0.01$. D. HSC3 cells were transfected with USP7 siRNA. Cell cycle-related and apoptosis-related genes were examined by WB at $48 \mathrm{~h}$. 
Caspase-3, cleaved Caspase-3 and Bax were all increased, whereas the apoptosis inhibiting proteins Bcl-2 and Bcl-XL were decreased in the siUSP7 group (Figure 2D). These data suggested that USP7 knockdown inhibited HSC3 cell proliferation by regulating cell cycle and cyclin-dependent kinase inhibitors-related proteins, thus promoting HSC3 cells apoptosis by upregulating pro-apoptotic related protein.

\section{Suppression of OSCC cell migration and invasion by USP7 knockdown}

The migration and invasion abilities were examined to investigate how USP7 may influence OSCC cells malignancy. Wound-healing assay was conducted to determine cell migration. The migration of HSC3 cells with siUSP7 was substantially reduced compared with blank and siRNA control groups (Figure 3A). Transwell assays showed that USP7 knockdown obviously suppressed OSCC cells migration and invasion compared to control groups (Figure 3B-C). The expression of migration and invasion related factors MMP2, MMP9 and VEGF were also decreased after USP7 knockdown in HSC3 cells (Figure 3D). These data were in line with previous results from immunohistochemistry staining in OSCC specimens, suggesting that the migration and invasion abilities were inhibited significantly after USP7 knockdown.

\section{USP7 promotes malignancy of OSCC cells via activating Akt/ERK signaling pathway}

The present data showed that USP7 could promote OSCC cell migration and invasion. But the underlying mechanism is not well defined. It is well known that epithelial-mesenchymal transition (EMT) is involved in OSCC cell invasion and metastasis (Cai et al., 2019). So the role of USP7 in EMT

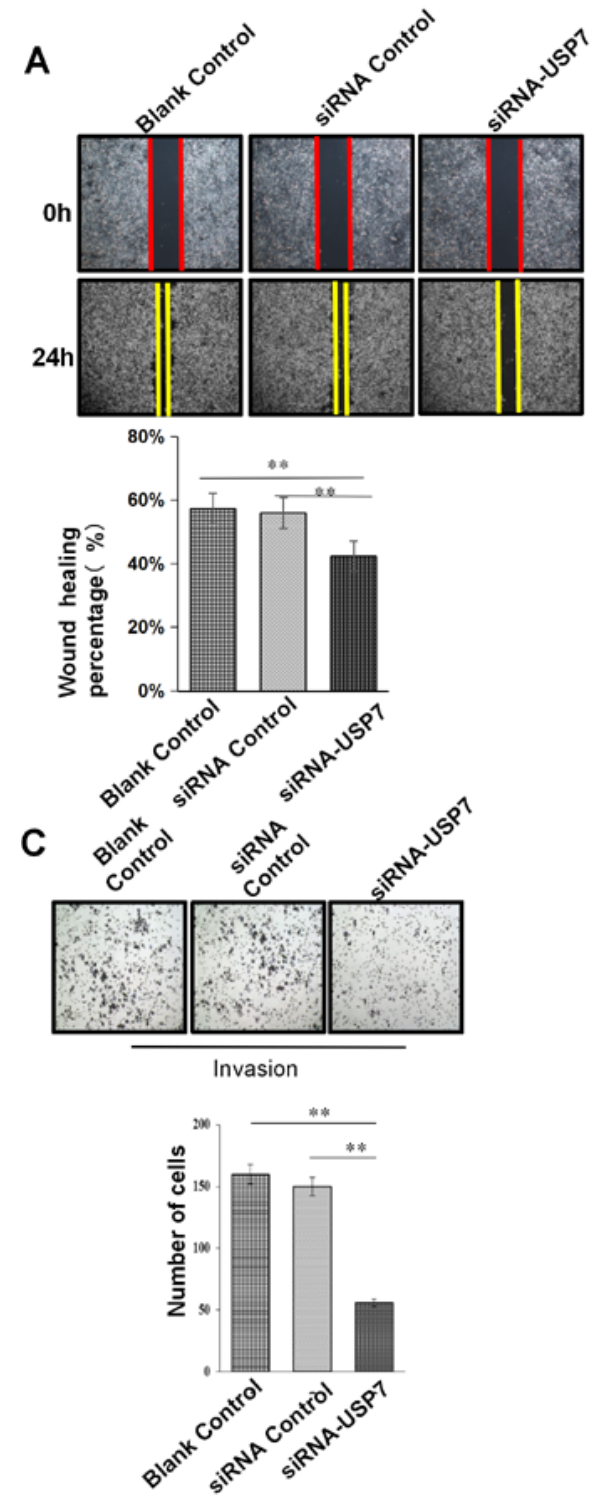

B
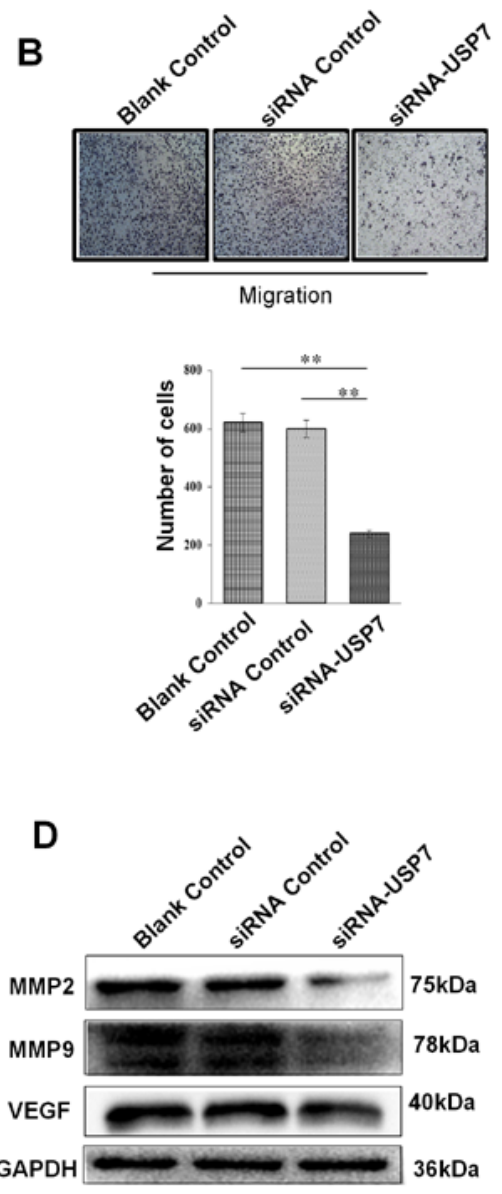

Figure 3 - USP7 knockdown suppresses migration and invasion of OSCC cells in vitro. A. Wound healing assay showed migrating ability was inhibited by USP7 knockdown in HSC3 cell line. B. Tranwell assay demonstrated that USP7 knockdown inhibited the migration ability of HSC3 cells. Statistics on the number of cell metastases after USP7 knockdown in HSC3 cells. C. Transwell test was performed to detect HSC3 cell invasive ability after USP7 knockdown. D. The relative gene expression of MMP2, MMP9, VEGF in HSC3 cells transfected with USP7 siRNA or siRNA negative control, blank control, were determined by WB analysis. GAPDH was used as a control. Data were presented as mean $\pm \mathrm{SEM}, * * P<0.01$. 
was examined. E-cadherin expression was upregulated and Vimentin, Snail, and Slug expression were all decreased in OSCC cells with USP7 knowdown (Figure 4). These data suggested that USP7 increased cell migration and invasion abilities by inducing EMT of OSCC cells.

Akt/ERK signaling pathway has been reported to be involved in OSCC proliferation, invasion and migration (Chiu et al., 2014; Chen et al., 2019). Here we investigated the effects of USP7 in the development of OSCC through Akt/ERK signaling pathway. In HSC3 cells with USP7 knockdown, p-Akt and p-ERK expression were all significantly decreased. These findings suggested that USP7 modulated malignancy of OSCC cells via activating Akt/ERK signaling pathway (Figure 4).

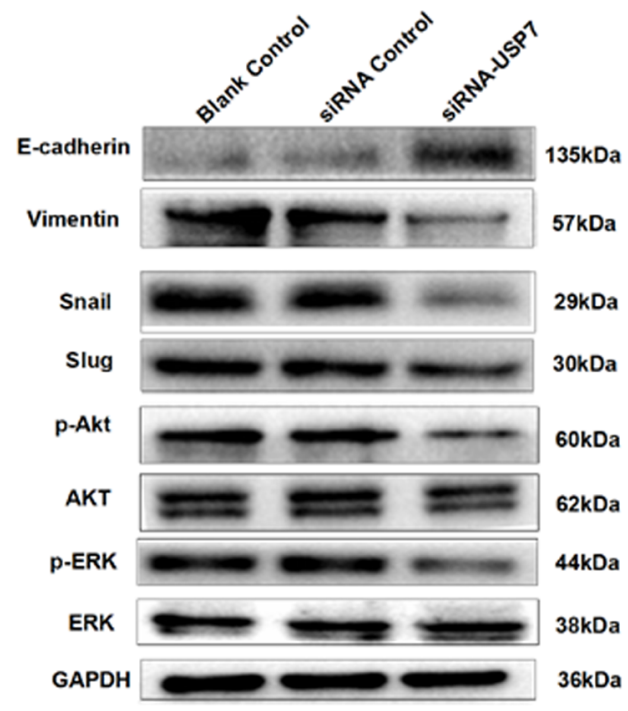

Figure 4

Figure 4 - USP7 induces EMT of OSCC cells via driving the Akt/ERK signaling pathway. The expression of E-cadherin, vimentin, Snail, Slug, ERK and AKT pathway proteins in HSC3 cells with USP7 siRNA or siRNA negative control, blank control were determined by WB. GAPDH expression was used as a control.

\section{Discussion}

USP7 expression was correlated with poor outcome of several cancers, such as non small cell lung cancer, large cell carcinoma, and lung squamous cell carcinoma (Masuya et al., 2006; Morra et al., 2015). The current study demonstrated that the expression of USP7 was relatively low in normal oral mucosa tissues adjacent to cancer tissues, but showed a high expression level in OSCC tissues, especially in poorly differentiated carcinomas (Figure 1 and Table 1). Notably, the expression of USP7 is positively correlation with Ki-67, MMP2, VEGF expression in OSCC cases (Table 2), USP7 expression was closely related to the degree of OSCC lymph node metastasis and histological differentiation (Table 3). Therefore, USP7 may serve as a novel and potential therapeutic target in OSCC.

The essential role of USP7 during cell cycle progression and apoptosis has been reported by other groups: i) USP7 knockdown leads to increased p53 expression and arrest of cell cycle (Cummins et al., 2004), ii) USP7 regulated various tumor-related factors expression (p53, Ki-67) in bladder cancer and prostate cancer (Song et al., 2008; Morra et al., 2019). So its expression is closely related to tumor cell proliferation. USP7 can stabilize Ki-67 protein through inhibiting its deubiquitination function (Zhang et al., 2016).

In the present study, the proliferation of HSC 3 cells was inhibited in OSCC cells with USP7 knockdown (Figure 2C); Cyclin A, Cyclin B, and Cyclin D1 proteins were decreased, whereas p21 and p27, the cyclin-dependent kinase inhibitors were all increased (Figure 2D); Caspase-3, cleaved Caspase-3 and $\mathrm{Bax}$ proteins were increased and the expression of $\mathrm{Bcl}-2$ and Bcl-XL, the apoptosis-inhibiting proteins were decreased (Figure 2D). These data indicate that USP7 was associated with the development of OSCC by inducing cell proliferation through activating cell cycle and inhibiting cell apoptosis.

On the other hand, it is widely known that USP7 overexpression in prostate cancer, ovarian cancer and liver cancer is highly associated with tumor metastasis and invasion (Nicholson and Suresh Kumar, 2011; Ma and Yu, 2016). Wound-healing assay and Transwell test have shown that OSCC cells transfected with USP7 siRNA could decrease migration and invasion abilities (Figure 3A-C). WB results showed that USP7 was positively associated to MMP2, MMP9 and VEGF expression in HSC3 cells. In OSCC cells with USP7 knockdown, MMP2, MMP9 and VEGF proteins were also decreased (Figure 3D).

It is well-known that the activation of the Akt/ERK pathway is frequently detected in OSCC (Mishima et al., 2002). Whether USP7 can directly participate in the regulation of these pathways is currently unclear. WB results showed that USP7 silencing decreased the expression level of p-Akt, p-ERK, Vimentin, Snail, Slug proteins (Figure 4), indicating that USP7 may affect the Akt/ERK pathways, thereby affect the EMT of OSCC.

In conclusion, the current study indicates that USP7 is weakly expressed in normal oral mucosa tissues but with a high expression in OSCC tissues. Its expression is positively associated to migration and invasion-related factors MMP2, MMP9 and VEGF. USP7 is closely related to the clinical pathological features, such as tumor histological differentiation and lymph node metastasis. In OSCC cells, USP7 was shown to promote cell proliferation, inhibit apoptosis, enhance cell migration and invasion, and activate the Akt/ERK pathway. These effects all contributed to the development of OSCC. Further investigating the specific substrate protein that are modified by USP7 could provide a new direction for exploring the progression of OSCC.

\section{Acknowledgments}

The study was supported by National Natural Science Foundation of China (grant no.[81660450]; [82060034]; [82100234]), Guangxi Natural Science Foundation Project (grant no.[2020GXNSFBA297004]), Guangxi University Middle-aged and Young Teachers' Basic Research Ability Improvement Project (grant no.[2020KY12014]), and Independent project of Guangxi Key Laboratory of Tumor Immunity and Microenvironment Regulation (grant no.[203030302007]). 


\section{Conflict of Interest}

The research was impartial and no conflict of interest was reported by authors.

\section{Author Contributions}

JMJ conceived the idea and designed experiments. GYQ provided the conceptual framework for the study. XJY performed the experiments. LHZ and JFY wrote the manuscript. SSM performed the data analysis, all authors read and approved the final version.

\section{References}

Bray F, Ferlay J, Soerjomataram I, Siegel RL, Torre LA and Jemal A (2018) Global cancer statistics 2018: GLOBOCAN estimates of incidence and mortality worldwide for 36 cancers in 185 countries. CA Cancer J Clin 68:394-424.

Cai H, Li J, Zhang Y, Liao Y, Zhu Y, Wang C and Hou J (2019) LDHA promotes oral squamous cell carcinoma progression through facilitating glycolysis and epithelial-mesenchymal transition. Front Oncol 9:1446.

Celentano A, Yap T, Paolini R, Yiannis C, Mirams M, Koo K, McCullough M and Cirillo N (2021) Inhibition of matrix metalloproteinase- 2 modulates malignant behaviour of oral squamous cell carcinoma cells. J Oral Pathol Med 50:323-332.

Chen Y-L, Liu K-J, Jang C-W, Hsu C-C, Yen Y-C, Liu Y-L, Chuang T-H, Wang S-H, Fu Y-K, Kuo C-C et al. (2019) ERK activation modulates cancer stemness and motility of a novel mouse oral squamous cell carcinoma cell line. Cancers (Basel) 12:61.

Chiu C-F, Bai L-Y, Kapuriya N, Peng S-Y, Wu C-Y, Sargeant AM, Chen MY and Weng J-R (2014) Antitumor effects of BI-D1870 on human oral squamous cell carcinoma. Cancer Chemother Pharmacol 73:237-247.

Colland F, Formstecher E, Jacq X, Reverdy C, Planquette C, Conrath S, Trouplin V, Bianchi J, Aushev VN, Camonis $\mathrm{J}$ et al. (2009) Small-molecule inhibitor of USP7/HAUSP ubiquitin protease stabilizes and activates p53 in cells. Mol Cancer Ther. 8:2286-2295.

Cummins JM, Rago C, Kohli M, Kinzler KW, Lengauer C and Vogelstein B (2004) Tumour suppression: disruption of HAUSP gene stabilizes p53. Nature 428:1 p following 486.

Everett RD, Meredith M, Orr A, Cross A, Kathoria M and Parkinson J (1997) A novel ubiquitin-specific protease is dynamically associated with the PML nuclear domain and binds to a herpesvirus regulatory protein. EMBO J 16:1519-1530.

Haddad RI and Shin DM (2008) Recent advances in head and neck cancer. N Engl J Med 359:1143-1154.

Jing Y, Yang Y, Hao F, Song Y, Zhang X, Zhang Y, Huang X, Hu Q and Ni Y (2018) Higher Ki67 expression in fibroblast like cells at invasive front indicates better clinical outcomes in oral squamous cell carcinoma patients. Biosci Rep Nov 38:BSR20181271.

Liu T, Liu J, Chen Q, Jin S, Mi S, Shao W, Kudo Y, Zeng S and Qi G (2019) Expression of USP22 and the chromosomal passenger complex is an indicator of malignant progression in oral squamous cell carcinoma. Oncol Lett 17:2040-2046.

Ma M and Yu N (2016) Ubiquitin-specific protease 7 expression is a prognostic factor in epithelial ovarian cancer and correlates with lymph node metastasis. Onco Targets Ther 9:1559-1569.

Mărgăritescu C, Pirici D, Simionescu C, Mogoantă L, Raica M, Stîngă A, Ciurea R, Stepan A, Stîngă A and Ribatti D (2009) VEGF and VEGFRs expression in oral squamous cell carcinoma. Rom J Morphol Embryol 50:527-548.
Mărgăritescu C, Pirici D, Stîngă A, Simionescu C, Raica M, Mogoantă L, Stepan A and Ribatti D (2010) VEGF expression and angiogenesis in oral squamous cell carcinoma: an immunohistochemical and morphometric study. Clin Exp Med 10:209-214.

Masuya D, Huang C, Liu D, Nakashima T, Yokomise H, Ueno M, Nakashima N and Sumitomo S (2006) The HAUSP gene plays an important role in non-small cell lung carcinogenesis through p53-dependent pathways. J Pathol 208:724-732.

Mishima K, Inoue K and Hayashi Y (2002) Overexpression of extracellular-signal regulated kinases on oral squamous cell carcinoma. Oral Oncol 38:468-474.

Morra F, Luise C, Merolla F, Poser I, Visconti R, Ilardi G, Paladino $\mathrm{S}$, Inuzuka H, Guggino G, Monaco R et al. (2015) FBXW7 and USP7 regulate CCDC6 turnover during the cell cycle and affect cancer drugs susceptibility in NSCLC. Oncotarget 6:12697-12709.

Morra F, Merolla F, Criscuolo D, Insabato L, Giannella R, Ilardi G, Cerrato A, Visconti R, Staibano S and Celetti A (2019) CCDC6 and USP7 expression levels suggest novel treatment options in high-grade urothelial bladder cancer. J Exp Clin Cancer Res 38:90.

Nicholson B and Suresh Kumar KG (2011) The multifaceted roles of USP7: new therapeutic opportunities. Cell Biochem Biophys 60:61-68.

Sarkari F, Sheng Y and Frappier L (2010) USP7/HAUSP promotes the sequence-specific DNA binding activity of p53. PLoS One 5:e13040.

Song MS, Salmena L, Carracedo A, Egia A, Lo-Coco F, TeruyaFeldstein J and Pandolfi PP (2008) The deubiquitinylation and localization of PTEN are regulated by a HAUSP-PML network. Nature 455:813-817.

Tang B, Liang X, Tang F, Zhang J, Zeng S, Jin S, Zhou L, Kudo $Y$ and Qi G (2015) Expression of USP22 and Survivin is an indicator of malignant behavior in hepatocellular carcinoma. Int J Oncol 47:2208-2216.

Wikner J, Gröbe A, Pantel K and Riethdorf S (2014) Squamous cell carcinoma of the oral cavity and circulating tumour cells. World J Clin Oncol 5:114-124.

Young M-J, Hsu K-C, Lin TE, Chang W-C and Hung J-J (2019) The role of ubiquitin-specific peptidases in cancer progression. $\mathrm{J}$ Biomed Sci 26:42.

Zeng Q, Li Z, Zhao X, Guo L, Yu C, Qin J, Zhang S, Zhang Y and Yang $X$ (2019) Ubiquitin-specific protease 7 promotes osteosarcoma cell metastasis by inducing epithelial-mesenchymal transition. Oncol Rep 41:543-551.

Zhang C, Lu J, Zhang Q-W, Zhao W, Guo J-H, Liu S-L, Wu Y-L, Jiang B and Gao F-H (2016) USP7 promotes cell proliferation through the stabilization of Ki-67 protein in non-small cell lung cancer cells. Int J Biochem Cell Biol 79:209-221.

Zhang WB, Wang Y, Mao C, Guo CB, Yu GY and Peng X (2019) Oral squamous cell carcinoma with metastasis to the parotid lymph node. Chin J Dent Res 22:175-179.

Zhou J, Wang J, Chen C, Yuan H, Wen X and Sun H (2018) USP7: Target validation and drug discovery for cancer therapy. Med Chem 14:3-18.

Jason LH, Rhonda KY, Laura WL, Steven DB, Raja RS, Ilan W, David BK, Zubair B, Daniel B, Ashley MCM. (2020) Abstracts from USCAP 2020: Head and neck pathology (1235-1315). Mod Pathol 33:1338-1408.

\section{Associate Editor: Ricardo G. Correa}

License information: This is an open-access article distributed under the terms of the Creative Commons Attribution License (type CC-BY), which permits unrestricted use, distribution and reproduction in any medium, provided the original article is properly cited. 